Pacific Journal of Mathematics

REGULAR EMBEDDINGS OF A GRAPH 


\title{
REGULAR EMBEDDINGS OF A GRAPH
}

\section{Hiroshi MaehaRA}

\begin{abstract}
In this paper we study embeddings of a graph $G$ in Euclidean space $R^{n}$ that are 'regular' in the following sense: given any two distinct vertices $u$ and $v$ of $G$, the distance between the corresponding points in $R^{n}$ equals $\alpha$ if $u$ and $v$ are adjacent, and equals $\beta$ otherwise. It is shown that for any given value of $s=\left(\beta^{2}-\alpha^{2}\right) / \beta^{2}$, the minimum dimension of a Euclidean space in which $G$ is regularly embeddable is determined by the characteristic polynomials of $G$ and $\vec{G}$.
\end{abstract}

1. Introduction. To embed a graph in Euclidean spaces with various restrictions, and to find the minimum dimension of the space for these embeddings, are interesting problems [1], [4], [5]. In this paper we consider a regular embedding of a graph.

An embedding of a graph $G$ in a Euclidean space $R^{n}$ is called a regular embedding of $G$ provided that, for any two distinct vertices $u$ and $v$ of $G$, the distance between the corresponding points in $R^{n}$ equals $\alpha$ if $u$ and $v$ are adjacent, and equals $\beta$ otherwise. The vertices of $G$ are mapped onto distinct points of $R^{n}$, but there is no restriction on the crossing of edges. The value $s=\left(\beta^{2}-\alpha^{2}\right) / \beta^{2}$ is called the parameter of the regular embedding. Let $\operatorname{dim}(G, s)$ denote the minimum number $n$ such that $G$ can be regularly embedded in $R^{n}$ with parameter $s$.

Consider, for example, the circuit graph $C_{5}$. For every regular embedding of $C_{5}$, it is seen that

$$
\frac{1}{2}(-\sqrt{5}-1) \leq s \leq \frac{1}{2}(\sqrt{5}-1)
$$

and

$$
\operatorname{dim}\left(C_{5}, s\right)= \begin{cases}2 & \text { if } s=\frac{1}{2}( \pm \sqrt{5}-1) \\ 4 & \text { otherwise. }\end{cases}
$$

The 'critical' embeddings of $C_{5}$ in $R^{2}$ with $s=\frac{1}{2}( \pm \sqrt{5}-1)$ are illustrated in Fig. 1.

Let $\phi(G ; x)$ denote the characteristic polynomial of a graph $G$ (that is, $\phi(G ; x)=|x \mathbf{I}-\mathbf{A}(G)|)$, and put

$$
\Phi(G ; x)=\phi(G ;-x)-(-1)^{g} \phi(\bar{G} ; x-1),
$$


where $g$ is the number of vertices of $G$, and $\bar{G}$ is the complement of $G$. Let $x^{-}$and $x^{+}$be, respectively, the minimum root and the maximum root of the polynomial $\Phi(G ; x)$. Suppose that $x^{-}<0$, and $1<x^{+}$. Then our results are stated as follows.

For every regular embedding of $G, 1 / x^{-} \leq s \leq 1 / x^{+}$and

$$
\operatorname{dim}\left(G, 1 / x^{*}\right)=g-1-\left(\text { the multiplicity of the root } x^{*}\right),
$$

where $x^{*}=x^{-}$or $x^{+}$. For other values of $s, \operatorname{dim}(G, s)=g-1$.
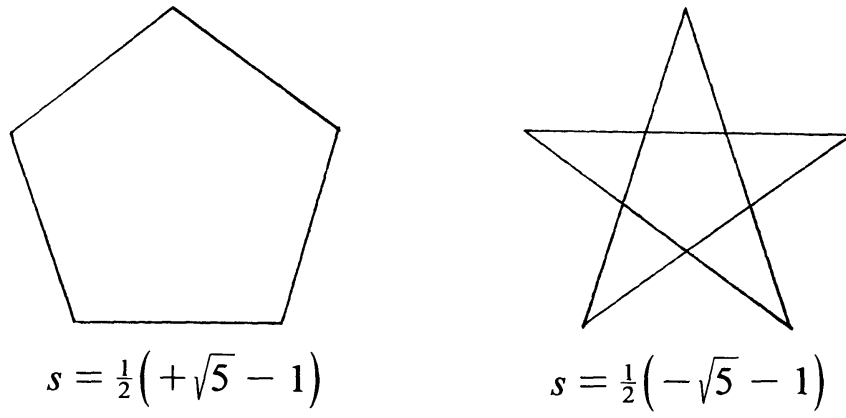

FIGURE 1

2. A theorem for isometric embeddings. We shall recall a theorem in distance geometry ([2], Ch. IV). Let $S=\left\{p_{0}, \ldots, p_{k}\right\}$ be a finite semimetric space with distance function $d$. The determinant

$$
\left|\begin{array}{ccccc}
0 & 1 & . & \cdots & 1 \\
1 & 0 & d_{01} & \cdots & d_{0 k} \\
\cdot & d_{10} & 0 & & \cdot \\
\vdots & \vdots & & \ddots & \vdots \\
1 & d_{k 0} & . & \cdots & 0
\end{array}\right| \quad d_{i j}:=d\left(p_{i}, p_{j}\right)^{2}
$$

is called the Cayley-Menger determinant of the semimetric space $(S, d)$, and is denoted by $D(S)$ or by $D\left(p_{0} \cdots p_{k}\right)$. Note that the value of the determinant does not depend on a labeling (ordering) $p_{0}, \ldots, p_{k}$ of the points of $S$.

If $S=\left\{p_{0}, \ldots, p_{k}\right\} \subset R^{n}, n \geq k$, then we denote by $\operatorname{Vol}(S)$ the $k$-dimensional volume of the simplex (perhaps degenerate) spanned by $S$. In this case, $\operatorname{Vol}(S)$ and the Cayley-Menger determinant of $S$ are related as follows:

$$
\operatorname{Vol}(S)^{2}=\frac{(-1)^{k+1}}{2^{k}(k !)^{2}} D(S) .
$$

For details, see Blumenthal [2], p. 98. 
A semimetric space $S$ is said to be irreducibly embeddable in $R^{n}$ provided that it is isometric to a subset of $R^{n}$ but not isometric to any subset of $R^{n-1}$.

THEOREM (Blumenthal [2]). A semimetric space $S$ is irreducibly embeddable in $R^{n}$ if and only if

(i) $S$ contains an $(n+1)$-tuple $p_{0}, \ldots, p_{n}$ such that

$$
\operatorname{sign} D\left(p_{0} \cdots p_{j}\right)=(-1)^{j+1} \quad(j=1, \ldots, n) ;
$$

(ii) for every pair $x, y$ of points of $S$,

$$
D\left(p_{0} \cdots p_{n}, x\right)=D\left(p_{0} \cdots p_{n}, y\right)=D\left(p_{0} \cdots p_{n}, x, y\right)=0 \text {. }
$$

3. The Cayley-Menger polynomial of a graph. A regular embedding of a graph $G$ with parameter $s$ is called, briefly, an $s$-embedding of $G$. To apply Blumenthal's theorem let us define a distance function $d_{s}$ on the vertex set $V(G)$ of $G$ by

$$
d_{s}(u, v)= \begin{cases}0 & \text { if } u=v \\ (1-s)^{1 / 2} & \text { if } u \text { and } v \text { are adjacent } \\ 1 & \text { otherwise }\end{cases}
$$

Then the Cayley-Menger determinant of the semimetric space $\left(V(G), d_{s}\right)$ is a polynomial in $s$, which we shall call the Cayley-Menger polynomial of $G$ and denote by $\operatorname{CM}(G ; s)$. For example, $\operatorname{CM}\left(K_{n} ; s\right)=(-1)^{n} n(1-s)^{n-1}$, and $\operatorname{CM}\left(\bar{K}_{n} ; s\right)=(-1)^{n} n$, where $K_{n}$ denotes the complete graph of order $n$.

Since there is a 0 -embedding of $G$ in a Euclidean space as a regular simplex of side-length 1 , we can restate Blumenthal's theorem in the following way. For any two graphs $G$ and $H$, let $H \subset G$ mean that $H$ is an induced subgraph of $G$.

THEOREM 1. There exists a t-embedding $(t<1)$ of a graph $G$ in $R^{n}$ if and only if there is $a G_{0} \subset G$ with $g_{0}(\leq n+1)$ vertices such that

(i) for any $F \subset G_{0}$, $\operatorname{sign} \mathrm{CM}(F ; t)=\operatorname{sign} \mathrm{CM}(F ; 0)$;

(ii) for any $G_{0} \subsetneq H \subset G, \mathrm{CM}(H ; t)=0$. In this case, $\operatorname{dim}(G, t)=g_{0}-1$.

Let $s^{+}(G)$ be the minimum positive root of the polynomial $\operatorname{CM}(G ; s)$, if it exists, and $\infty$ otherwise. For example, $s^{+}\left(K_{2}\right)=1$, and $s^{+}\left(\bar{K}_{n}\right)=\infty$. Let $s^{-}(G)$ be the maximum negative root of $\operatorname{CM}(G ; s)$, if it exists, and $-\infty$ otherwise. 
LEMMA 1. For $H \subset G, s^{-}(H) \leq s^{-}(G)<s^{+}(G) \leq s^{+}(H)$.

Proof. We shall only show that $s^{+}(G) \leq s^{+}(H)$. Let $s_{0}$ be the minimum value of $s^{+}(F)$ for $F \subset G$. It is sufficient to show that $s_{0} \geq$ $s^{+}(G)$. If $s_{0}=\infty$ then clearly $s_{0}=s^{+}(G)=\infty$. Suppose $s_{0}<\infty$ and $\mathrm{CM}\left(F_{0} ; s_{0}\right)=0$ for some $F_{0} \subset G$. In this case, $s_{0} \leq 1$, because $s^{+}\left(K_{2}\right)=1$. Since $\operatorname{sign} \mathrm{CM}(F ; s)=\operatorname{sign} \mathrm{CM}(F ; 0)$ for $F \subset G$ and for $0 \leq s<s_{0}$, it follows from Theorem 1 that for every $0 \leq s<s_{0}$, there is an $s$-embedding $f_{s}: G \rightarrow R^{n}$ of $G$ where $n+1 \geq g:=|V(G)|$, the cardinality of the vertex set $V(G)$ of $G$. Since $\operatorname{Vol}\left(f_{s}\left(V\left(F_{0}\right)\right)\right)^{2}$ is the product of $\operatorname{CM}\left(F_{0} ; s\right)$ by a constant, and $\operatorname{CM}\left(F_{0} ; s_{0}\right)=0$, we have

$$
\operatorname{Vol}\left(f_{s}\left(V\left(F_{0}\right)\right)\right) \rightarrow 0 \quad \text { as } s \rightarrow s_{0} .
$$

Hence we have

$$
\operatorname{Vol}\left(f_{s}(V(G))\right) \rightarrow 0 \quad \text { as } s \rightarrow s_{0} .
$$

Then by the continuity, $\operatorname{CM}\left(G ; s_{0}\right)=0$, and hence $s_{0} \geq s^{+}(G)$.

Note that if $G$ contains at least one edge, then $s^{+}(G) \leq 1$.

TheOREM 2. For every $s^{-}(G)<s<\min \left(s^{+}(G), 1\right)$, there is an $s$-embedding of $G$, and $\operatorname{dim}(G, s)=g-1$. If $-\infty<s^{*}(G)<1$ then there is an $s^{*}(G)$-embedding of $G$, where $s^{*}(G)=s^{-}(G)$ or $s^{+}(G)$.

Proof. We shall only prove the existence of an $s^{+}(G)$-embedding of $G$, provided that $s^{+}(G)<1$. Let $H$ be a maximal induced subgraph of $G$ such that $\mathrm{CM}\left(H ; s^{+}(G)\right) \neq 0$. Then

(i) if $F \subset H$ then $s^{+}(G)<s^{+}(H) \leq s^{+}(F)$, and hence

$$
\operatorname{sign} \mathrm{CM}\left(F ; s^{+}(G)\right)=\operatorname{sign} \mathrm{CM}(F ; 0) \text {; }
$$

(ii) if $H \subsetneq F \subset G$, then $\mathrm{CM}\left(F ; s^{+}(G)\right)=0$ by the maximality of $H$. Hence there is an $s^{+}(G)$-embedding of $G$, by Theorem 1 .

4. Calculation of $\operatorname{CM}(G ; s)$. Let $\mathbf{I}_{r}$ and $\mathbf{J}_{r}$ denote, respectively, the identity $r \times r$ matrix and $r \times r$ matrix each entry of which is 1 . (In the following, the subscripts are often omitted.) Put $\mathbf{K}_{r}=\mathbf{J}_{r}-\mathbf{I}_{r}$ and

$$
\mathbf{B}(G)=\left[\begin{array}{ccc}
0 & \cdots & 0 \\
\vdots & \mathbf{A}(G) & \\
0 & &
\end{array}\right],
$$

where $\mathbf{A}(G)$ is the adjacency matrix of $G$, and put $g=|V(G)|$. Then, by 
the definition of $\mathrm{CM}(G ; s)$,

$$
\mathrm{CM}(G ; s)=\left|\mathbf{K}_{g+1}-s \mathbf{B}(G)\right|=|s \mathbf{K}|\left|(1 / s) \mathbf{I}-\mathbf{K}^{-1} \mathbf{B}(G)\right| .
$$

Since $\mathbf{K}^{-1}=(1 / \mathrm{g}) \mathbf{J}-\mathbf{I}$,

$$
\begin{aligned}
\mathbf{K}^{-1} \mathbf{B}(G) & =(1 / g) \mathbf{J B}(G)-\mathbf{B}(G) \\
& =\left[\begin{array}{cccc}
0 & d_{1} / g & \cdots & d_{g} / g \\
\vdots & & & \vdots \\
0 & d_{1} / g & \cdots & d_{g} / g
\end{array}\right]-\mathbf{B}(G),
\end{aligned}
$$

where $d_{i}$ is the sum of entries in the $i$ th column of $\mathbf{A}(G)$. In the matrix $x \mathbf{I}-\mathbf{K}^{-1} \mathbf{B}(G)$, by subtracting the top row from other rows, we have

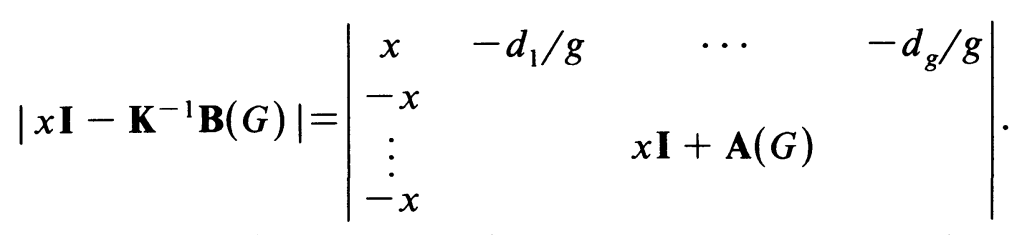

On the right-hand side, adding to the top row the product of the $i$ th row by $1 / g, i=2, \ldots, g+1$, we have

$$
\begin{aligned}
& \left|x \mathbf{I}-\mathbf{K}^{-1} \mathbf{B}(G)\right|=\left|\begin{array}{lccc}
0 & x / g & \cdots & x / g \\
-x & & & \\
\vdots & & & \\
-x & \mathbf{I}(G) &
\end{array}\right| \\
& =-x^{2} / g\left|\begin{array}{cccc}
0 & 1 & \cdots & 1 \\
1 & & \\
\vdots & & x \mathbf{I}+\mathbf{A}(G)
\end{array}\right|
\end{aligned}
$$

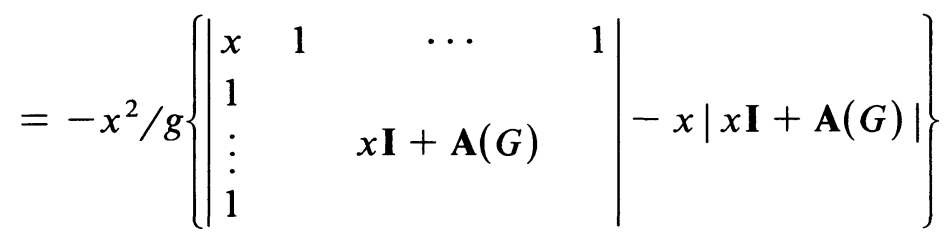

$$
\begin{aligned}
& =-x^{2} / g\left\{\left|x \mathbf{I}_{g+1}+\mathbf{A}\left(G+K_{1}\right)\right|-x|x \mathbf{I}+\mathbf{A}(G)|\right\}
\end{aligned}
$$

(where $G+K_{1}$ is the join of $G$ and $K_{1}$, defined by $\overline{G+K_{1}}=\bar{G} \cup \bar{K}_{1}$ )

$$
\begin{aligned}
& =-x^{2} / g\left\{(-1)^{g+1}\left|(-x) \mathbf{I}-\mathbf{A}\left(G+K_{1}\right)\right|\right. \\
& \left.\quad+(-1)^{g+1} x|(-x) \mathbf{I}-\mathbf{A}(G)|\right\} \\
& \left.=(-1)^{g} x^{2} / g\left\{\phi\left(G+K_{1}\right) ;-x\right)+x \phi(G ;-x)\right\} .
\end{aligned}
$$


Using Cvetković's theorem ([3], p. 57):

$$
\begin{aligned}
\phi\left(G_{1}+\right. & \left.G_{2} ; x\right) \\
= & (-1)^{g_{2}} \phi\left(G_{1} ; x\right) \phi\left(\bar{G}_{2} ;-x-1\right) \\
& +(-1)^{g_{1}} \phi\left(G_{2} ; x\right) \phi\left(\bar{G}_{1} ;-x-1\right) \\
& -(-1)^{g_{1}+g_{2}} \phi\left(\bar{G}_{1} ;-x-1\right) \phi\left(\bar{G}_{2} ;-x-1\right), \quad g_{i}=\left|V\left(G_{i}\right)\right| .
\end{aligned}
$$

After a brief calculation, we have

$$
\left|x \mathbf{I}-\mathbf{K}^{-1} \mathbf{B}(G)\right|=(-1)^{g} x^{2} / g\left\{\phi(G ;-x)-(-1)^{g} \phi(\bar{G} ; x-1)\right\} .
$$

Since $\left|(1 / x) \mathbf{K}_{g+1}\right|=(-1)^{g} g(1 / x)^{g+1}$, we have the following:

\section{THEOREM 3.}

$$
\mathrm{CM}(G ; 1 / x)=(1 / x)^{g-1}\left\{\phi(G ;-x)-(-1)^{g} \phi(\bar{G} ; x-1)\right\} .
$$

5. Bounds on the parameter $s$. Put

$$
\Phi(G ; x)=\phi(G ;-x)-(-1)^{g} \phi(\bar{G} ; x-1),
$$

where $g$ is the number of vertices of $G$. Then Theorem 3 says

$$
\mathrm{CM}(G ; s)=s^{g-1} \Phi(G ; 1 / s) .
$$

Note that $s_{0} \neq 0$ is a root of $\operatorname{CM}(G ; s)$ if and only if $1 / s_{0}$ is a root of $\Phi(G ; x)$. Thus we have the following theorem:

THEOREM 4. The polynomial $\Phi(G ; x)$ has a positive root if and only if $s^{+}(G)<\infty$. In this case, $1 / s^{+}(G)$ is the maximum root of $\Phi(G ; x)$. The polynomial $\Phi(G ; x)$ has a negative root if and only if $s^{-}(G)>-\infty$. In this case, $1 / s^{-}(G)$ is the minimum root of $\Phi(G ; x)$.

Now let $V(G)=\left\{v_{1}, \ldots, v_{g}\right\}, g \geq 2$, and put

$$
G_{i_{1} \cdots i_{k}}=G-v_{i_{1}}-\cdots-v_{i_{k}}, \quad k \leq g-1 \text {. }
$$

LEMMA 2.

$$
\frac{d^{k}}{d x^{k}} \Phi(G ; x)=(-1)^{k} k ! \sum_{\left\{i_{1} \cdots i_{k}\right\}} \Phi\left(G_{i_{1} \cdots i_{k}} ; x\right),
$$

where the summation extends over all $k$-subsets of $\{1, \ldots, g\}$. 
Proof. Since

$$
\frac{d}{d x} \phi(G ; x)=\sum_{i=1}^{i=g} \phi\left(G_{i} ; x\right)
$$

(see [6], p. 331), we have

$$
\begin{aligned}
\frac{d}{d x} \Phi(G ; x) & =\frac{d}{d x}\left\{\phi(G ;-x)-(-1)^{g} \phi(\bar{G} ; x-1)\right\} \\
& =-\sum\left\{\phi\left(G_{i} ;-x\right)-(-1)^{g} \phi\left(\bar{G}_{i} ; x-1\right)\right\} \\
& =-\sum \Phi\left(G_{i} ; x\right) .
\end{aligned}
$$

Differentiating repeatedly, we have the lemma.

LEMMA 3. If $-\infty<s^{*}(G)<\infty$, and the multiplicity of the root $x^{*}=1 / s^{*}(G)$ of $\Phi(G ; x)$ equals $k+1$, then

$$
\Phi\left(G_{i_{1} \ldots i} ; x^{*}\right)=0 \quad \text { for } j \leq k,\left\{i_{1}, \ldots, i_{j}\right\} \subset\{1, \ldots, g\},
$$

where $s^{*}(G)=s^{-}(G)$ or $s^{+}(G)$.

Proof. Since $\Phi^{(j)}\left(G ; x^{*}\right)=0$ for $j \leq k$ it follows from the above lemma that

$$
(-1)^{j} j ! \sum \Phi\left(G_{i_{1} \ldots i} ; x^{*}\right)=0 .
$$

Since there is an $s$-embedding of $G$ for every $s^{-}(G)<s<s^{+}(G)$, it follows by the continuity that

$$
\operatorname{sign} \mathrm{CM}\left(G_{i_{1} \cdots i_{j}} ; s^{*}(G)\right)=(-1)^{g-j} \text { or } 0 .
$$

Since $\operatorname{CM}\left(G_{i_{1} \cdots i_{1}} ; s^{*}(G)\right)=s^{*}(G)^{g-j-1} \Phi\left(G_{i_{1} \cdots i} ; x^{*}\right)$, it follows that the non-zero term of the left-hand side of (\#) must have the same sign, which is impossible. Hence $\Phi\left(G_{i_{1} \cdots i_{j}} ; x^{*}\right)=0$.

THEOREM 5. If there is a t-embedding of $G$ then

$$
s^{-}(G) \leq t \leq s^{+}(G) \text {. }
$$

Proof. It is clear that the theorem holds true for graphs with fewer vertices than three. Assume that there exists a graph for which the theorem does not hold, and let $H$ be one of such graphs which is minimal in the number of vertices. Then there is a $t$-embedding of $H$ such that 
$t<s^{-}(H)$ or $s^{+}(H)<t$. Suppose that $s^{+}(H)<t$. (The case $t<s^{-}(H)$ is similar, and is omitted.) Let $V(H)=\left\{v_{1}, \ldots, v_{h}\right\}$, and put $H_{i}=H-v_{i}$, $i=1, \ldots, h ; x^{+}=1 / s^{+}(H)$. Then $x^{+}$is the maximum root of $\Phi(H ; x)$ and $1 / t<x^{+}$. By the minimality of $H, t \leq s^{+}\left(H_{i}\right), i=1, \ldots, h$.

Now we show that $x^{+}$is a simple root of $\Phi(H ; x)$. If $x^{+}$is a multiple root, then $\Phi\left(H_{i} ; x^{+}\right)=0$ by Lemma 3 , which implies that $s^{+}\left(H_{i}\right)=$ $s^{+}(H)<t$, a contradiction. Thus $x^{+}$must be a simple root of $\Phi(H ; x)$.

Since $\Phi(H ; x)$ changes sign when $x$ passes through $x^{+}$, a simple root, $\mathrm{CM}(H ; s)$ also changes sign when $s$ passes through $s^{+}(H)$. Since $\operatorname{sign} \mathrm{CM}(H ; t)=\operatorname{sign} \mathrm{CM}(H ; 0)$ or $\mathrm{CM}(H ; t)=0$ (because there is a $t$-embedding of $H$ ), and $s^{+}(H)<t$, there must be a root $s_{1}$ of $\operatorname{CM}(H ; s)$ such that $s^{+}(H)<s_{1} \leq t$. Thus $\Phi(H ; x)$ has a root $x_{1}=1 / s_{1}$ such that $1 / t \leq x_{1}<x^{+}$. Then, by Rolle's theorem, there is a $\xi, x_{1}<\xi<x^{+}$, such that $\Phi^{\prime}(H ; \xi)=0$. But since $1 / \xi<1 / x_{1} \leq t \leq s^{+}\left(H_{i}\right)$, there is a $(1 / \xi)$ embedding of $H_{i}$, and $\Phi\left(H_{i} ; \xi\right)$ is non-zero and has the same sign for every $i$. This contradicts the fact that $0=\Phi^{\prime}(H ; \xi)=-\Sigma \Phi\left(H_{i} ; \xi\right)$.

6. The dimension of a critical embedding. Let $G$ be a graph with vertex set $V(G)=\left\{v_{1}, \ldots, v_{g}\right\}$, and put

$$
G_{i_{1} \cdots i_{j}}=G-v_{i_{1}}-\cdots-v_{i_{j}}, \quad j<g .
$$

THEOREM 6. If $-\infty<s^{*}(G)<1$, and the multiplicity of the root $x^{*}=1 / s^{*}(G)$ of $\Phi(G ; x)$ equals $k$, then

$$
\operatorname{dim}\left(G, s^{*}(G)\right)=g-k-1,
$$

where $s^{*}(G)=s^{-}(G)$ or $s^{+}(G)$.

Proof. Since

$$
0 \neq \Phi^{(k)}\left(G ; x^{*}\right)=(-1)^{k} k ! \sum_{\left\{i_{1} \cdots i_{k}\right\}} \Phi\left(G_{i_{1} \cdots i_{k}} ; x^{*}\right),
$$

there is a $\left\{j_{1}, \ldots, j_{k}\right\}$ such that $\Phi\left(G_{j_{1} \ldots j_{k}} ; x^{*}\right) \neq 0$. By Lemma 1 , it follows easily that if $F \subset G_{j_{1} \cdots j_{k}}$ then

$$
\operatorname{sign} \operatorname{CM}\left(F ; s^{*}(G)\right)=\operatorname{sign} \operatorname{CM}(F ; 0) .
$$

Using Lemma 3, it follows that if $G_{j_{1} \cdots j_{k}} \subsetneq H \subset G$ then $\operatorname{CM}\left(H ; s^{*}(G)\right)=$ 0 . Hence $\operatorname{dim}\left(G ; s^{*}(G)\right)=g-k-1$, by Theorem 1 . 
7. On regular graphs. If $G$ is a regular $\rho$-valent graph with $g$ vertices, then by Sachs' theorem ([6], p. 56),

$$
\phi(\bar{G} ; x)=(-1)^{g} \frac{x+\rho+1-g}{x+\rho+1} \phi(G ;-x-1) .
$$

Hence we have

$$
\Phi(G ; x)=\frac{g}{x+\rho} \phi(G ;-x)
$$

Let $\lambda_{1} \geq \lambda_{2} \geq \cdots \geq \lambda_{g}$ be the eigenvalues of $\mathbf{A}(G)$. Then $\lambda_{1}=\rho$ is a simple root of $\phi(G ; x)$, and $\lambda_{g}<0$. Therefore

$$
s^{+}(G)=-1 / \lambda_{g}
$$

and

$$
s^{-}(G)= \begin{cases}-1 / \lambda_{2} & \text { if } \lambda_{2}>0 \\ -\infty & \text { otherwise }\end{cases}
$$

EXAMPLE. Let $G$ be the Petersen graph. The characteristic polynomial of $G$ is $(x-3)(x-1)^{5}(x+2)^{4}$. Hence $s^{+}(G)=1 / 2, s^{-}(G)=-1$, and

$$
\operatorname{dim}(G, s)= \begin{cases}4, & s=-1, \\ 5, & s=1 / 2, \\ 9, & -1<s<1 / 2\end{cases}
$$

Acknowledgement. I thank Timothy F. Havel of the University of California for informing me of several literature references and for interesting me in the study of distance geometry.

\section{REFERENCES}

[1] B. Alspach and M. Rosenfeld, On embedding triangle-free graphs in unit spheres, Discrete Math., 19 (1977), 103-111.

[2] L. M. Blumenthal, Theory and Applications of Distance Geometry, Chelsea, New York (1970).

[3] D. Cvetkovic̀, M. Doob and H. Sachs, Spectra of Graphs, Academic Press, New York (1980).

[4] P. Erdős, F. Harary and W. T. Tutte, On the dimension of a graph, Mathematika, 12 (1965), 118-122. 
[5] F. S. Roberts, On the boxicity and cubicity of a graph, in Recent Progress in Combinatorics (ed. by W. T. Tutte) Academic Press, New York (1969), pp. 301-310.

[6] A. J. Schwenk and R. J. Wilson, On the Eigenvalue of a Graph, in Selected Topics in Graph Theory (ed. by L. W. Beineke and R. J. Wilson), Academic Press, New York (1978).

Received September 10, 1981.

RYUKYU UNIVERSITY

NAHA, OKINAWA, JAPAN 


\title{
PACIFIC JOURNAL OF MATHEMATICS \\ EDITORS
}

\author{
DONALD BABBITT (Managing Editor) \\ University of California \\ Los Angeles, CA 90024 \\ Hugo Rossi \\ University of Utah \\ Salt Lake City, UT 84112 \\ C. C. Moore and Arthur Ogus \\ University of California \\ Berkeley, CA 94720
}

\author{
J. DugundiI \\ Department of Mathematics \\ University of Southern California \\ Los Angeles, CA 90089-1113
}

R. FINN and H. SAMELSON

Stanford University

Stanford, CA 94305

\begin{tabular}{lllll}
\multicolumn{3}{c}{ ASSOCIATE EDITORS } \\
R. ARENS & $\begin{array}{lll}\text { E. F. BeCKendach } \\
(1906-1982)\end{array}$ & B. H. NeumanN & F. Wolf & K. Yoshida
\end{tabular}

\begin{tabular}{ll}
\multicolumn{2}{c}{ SUPPORTING INSTITUTIONS } \\
UNIVERSITY OF ARIZONA & UNIVERSITY OF OREGON \\
UNIVERSITY OF BRITISH COLUMBIA & UNIVERSITY OF SOUTHERN CALIFORNIA \\
CALIFORNIA INSTITUTE OF TECHNOLOGY & STANFORD UNIVERSITY \\
UNIVERSITY OF CALIFORNIA & UNIVERSITY OF HAWAII \\
MONTANA STATE UNIVERSITY & UNIVERSITY OF TOKYO \\
UNIVERSITY OF NEVADA, RENO & UNIVERSITY OF UTAH \\
NEW MEXICO STATE UNIVERSITY & WASHINGTON STATE UNIVERSITY \\
OREGON STATE UNIVERSITY & UNIVERSITY OF WASHINGTON
\end{tabular}

The Supporting Institutions listed above contribute to the cost of publication of this Journal, but they are not owners or publishers and have no responsibility for its content or policies.

Mathematical papers intended for publication in the Pacific Journal of Mathematics should be in typed form or offset-reproduced (not dittoed), double spaced with large margins. Please do not use built up fractions in the text of the manuscript. However, you may use them in the displayed equations. Underline Greek letters in red, German in green, and script in blue. The first paragraph must be capable of being used separately as a synopsis of the entire paper. In particular it should contain no bibliographic references. Please propose a heading for the odd numbered pages of less than 35 characters. Manuscripts, in triplicate, may be sent to any one of the editors. Please classify according to the scheme of Math. Reviews, Index to Vol. 39. Supply name and address of author to whom proofs should be sent. All other communications should be addressed to the managing editor, or Elaine Barth, University of California, Los Angeles, California 90024.

There are page-charges associated with articles appearing in the Pacific Journal of Mathematics. These charges are expected to be paid by the author's University, Government Agency or Company. If the author or authors do not have access to such Institutional support these charges are waived. Single authors will receive 50 free reprints; joint authors will receive a total of 100 free reprints. Additional copies may be obtained at cost in multiples of 50 .

The Pacific Journal of Mathematics is issued monthly as of January 1966. Regular subscription rate: $\$ 132.00$ a year (6 Vol., 12 issues). Special rate: $\$ 66.00$ a year to individual members of supporting institutions.

Subscriptions, orders for numbers issued in the last three calendar years, and changes of address should be sent to Pacific Journal of Mathematics, P.O. Box 969, Carmel Valley, CA 93924, U.S.A. Old back numbers obtainable from Kraus Periodicals Co., Route 100, Millwood, NY 10546.

The Pacific Journal of Mathematics ISSN 0030-8730 is published monthly by the Pacific Journal of Mathematics at P.O. Box 969, Carmel Valley, CA 93924. Application to mail at Second-class postage rates is pending at Carmel Valley, California, and additional mailing offices. Postmaster: Send address changes to Pacific Journal of Mathematics, P. O. Box 969, Carmel Valley, CA 93924.

PUBLISHED BY PACIFIC JOURNAL OF MATHEMATICS, A NON-PROFIT CORPORATION

Copyright $\odot 1983$ by Pacific Journal of Mathematics 


\section{Pacific Journal of Mathematics}

Vol. 107, No. $2 \quad$ February, 1983

Driss Abouabdillah, Topologies de corps $A$ linéaires $\ldots \ldots \ldots \ldots \ldots . \ldots 257$

Patrick Robert Ahern, On the behavior near a torus of functions

holomorphic in the ball $\ldots \ldots \ldots \ldots \ldots \ldots \ldots \ldots \ldots \ldots \ldots \ldots \ldots . \ldots 267$

Donald Werner Anderson, There are no phantom cohomology operations

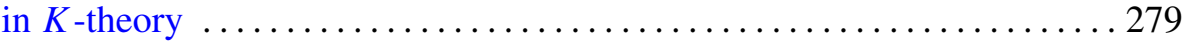

Peter Bloomfield, Nicolas P. Jewell and Eric Hayashi, Characterizations of

completely nondeterministic stochastic processes . ............. 307

Sydney Dennis Bulman-Fleming and K. McDowell, Absolutely flat

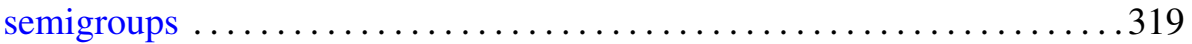

C. Debiève, On a Radon-Nikodým problem for vector-valued measures . . . 335

Dragomir Z. Djokovic, Products of positive reflections in real orthogonal

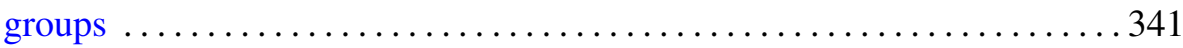

Thomas Farmer, The dual of the nilradical of the parabolic subgroups of

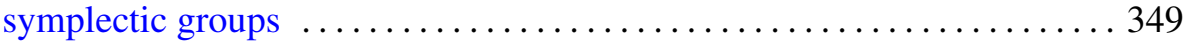

Gary R. Greenfield, Uniform distribution in subgroups of the Brauer group of an algebraic number field $\ldots \ldots \ldots \ldots \ldots \ldots \ldots \ldots \ldots \ldots \ldots . \ldots \ldots$

Paul Daniel Hill, When $\operatorname{Tor}(A, B)$ is a direct sum of cyclic groups $\ldots \ldots \ldots 383$

Hiroshi Maehara, Regular embeddings of a graph $\ldots \ldots \ldots \ldots \ldots \ldots \ldots 3$

Nikolaos S. Papageorgiou, Nonsmooth analysis on partially ordered vector spaces. I. Convex case . .............................4 403

Louis Jackson Ratliff, Jr., Powers of ideals in locally unmixed Noetherian

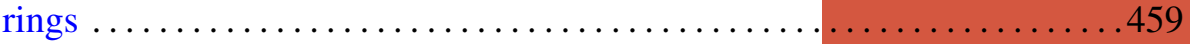

F. Dennis Sentilles and Robert Francis Wheeler, Pettis integration via the Stonian transform .......................... 